\title{
INFANT "SURPRISE" EXPRESSIONS AS COORDINATIVE MOTOR STRUCTURES*
}

By: Linda A. Camras, Linda Lambrecht, and George F. Michel

Camras, LA, Lambrecht, L \& Michel, GF. Infant "surprise" expressions as coordinative motor structures. Journal of Nonverbal Behavior. 1996; 20:183-195.

Made available courtesy of Springer Verlag:

http://www.springer.com/psychology/personality+\%26+social+psychology/journal/10919

\section{The original publication is available at www.springerlink.com}

\section{***Note: Figures may be missing from this format of the document}

\begin{abstract}
:
This study found that the facial action of moderately or widely opening the mouth is accompanied by brow raising in infants, thus producing "surprise" expressions in non-surprise situations. Infants (age $=5$ months and 7 months) were videotaped as they were presented with toys that they often grasped and brought to their mouths. Episodes of mouth opening were identified and accompanying brow, nose, and eyelid movements were coded. Results indicated that mouth opening is selectively associated with raised brows rather than to other brow movements. Trace levels of eyelid raising also tended to accompany this facial configuration. The findings are discussed in terms of a dynamical systems theory of facial behavior and suggest that facial expression cannot be used as investigators' sole measure of "surprise" in infants.
\end{abstract}

\section{Article:}

Numerous investigations (Ekman, 1982, 1989) have indicated that at least six distinct facial configurations are identified with specific discrete emotions by raters from a variety of Western and non-Western cultures. Despite recent challenges (Russell, 1994), these studies have provided some of the strongest and most widely replicated findings in the psychological literature (Ekman, 1994).

Following the initial demonstrations of cross-cultural recognition, a number of reasonable proposals were advanced regarding the natural occurrence of these facial configurations and their relationship to emotion throughout the course of development. Regarding adults, several emotion theorists (Buck, 1988; Ekman, 1972; Izard, 1977) proposed that these facial configurations were "direct readouts" of emotion. That is, these expressions would be automatically produced by the experiencer unless he or she inhibited them in accord with cultural or personal display rules. Extending this line of reasoning to include infants and children, one influential developmental theory (Differential Emotions Theory, Izard \& Malatesta, 1987) proposed that adultlike facial expressions emerge in infancy and represent discrete emotions from the time of their initial appearance. Indeed, because infants under one year of age were assumed not to inhibit their expressive behavior, facial expressions could be used as an independent and reliable index of infants' emotional experience.

Despite its theoretical and pragmatic appeal, empirical investigations of adult expressive behavior have failed to produce consistent evidence for the "direct read-out" hypothesis of emotional expression (see Camras, Holland, $\&$ Patterson, 1993, for review). For example, subjects viewing emotion-laden slides or films produce little expressive behavior unless they are asked to verbally describe the stimulus (Wagner, Buck, \& Winterbotham, 1993). Thus, several theorists (Ekman, 1992; Izard, 1991) currently acknowledge that the relationship between adults' emotional experience and expressive output may require reconsideration.

\footnotetext{
* This research was conducted as part of the second author's undergraduate honors program project and was supported in part by a grant from the NICHHD \#1R01 HD 22399-A3 awarded to G. F. Michel.
} 
Regarding infants, the direct-readout hypothesis has been challenged by a number of researchers (Barrett \& Campos, 1987; Lewis \& Michalson, 1983; Sroufe, 1979) who argue that facial expressions initially either are not tied to an emotion or are tied to diffuse, relatively undifferentiated hedonic states. For example, the REMstate smiles of neonates are considered to be nonemotional by many investigators (Emde, Gaensbauer, \& Harmon, 1976). The facial configuration of "anger" described by Izard (Izard, Dougherty, \& Hembree, 1983) has been interpreted as an expression of more diffuse negative emotion (i.e., "distress") by others (Camras, 1992; Oster, Hegley, \& Nagel, 1992). These alternative interpretations do not represent a theoretical retreat to viewing infant facial behavior as disorganized. or random. Rather, infant facial expressions are proposed to be systematically related to causal factors other than discrete, adult-like emotions.

A somewhat different alternative interpretation was proposed by Michel, Camras, and Sullivan (1992) for select variants of the infant "interest" expression. As described in Izard's AFFEX coding system (Izard et al., 1983), the family of interest expressions includes several diverse facial configurations. These include either knit (i.e., slightly contracted) brows or raised brows accompanied by either a relaxed open mouth or puckered or pursed lips. Michel et al. (1992) proposed that nonemotion factors might determine whether knit brows or raised brows would be produced by an "interested" infant and indeed would cause these movements to be produced even in situations in which the emotion of interest might not be present.

As the basis for their proposal, Michel et al. (1992) drew upon dynamical systems theory (Kelso, 1981; Kugler, Kelso, \& Turvey, 1982; Schoner \& Kelso, 1988), a recently developed framework for viewing the organization of action that has proved highly useful to investigators of infant motor development (Fogel \& Thelen, 1987; Michel, 1991; Thelen, Kelso, \& Fogel, 1987; Thelen \& Ulrich, 1991). Dynamical systems theory was developed in part as a response to Bernstein's (1967) problem: how does one account for the control of complex motor patterns that are enacted with an almost infinite number of minor variations (e.g., walking upon uneven ground)? Dynamical systems theory proposes that complex patterns of motor behavior are not completely determined by a central executive program since such a "command agent" would be overburdened by the need to accommodate for an almost infinite number of minor variations in the muscle movements comprising the action sequence. According to dynamical systems theory, the organization of complex actions is determined (largely or in part) by lower-order "coordinative motor structures" (Kelso \& Scholz, 1986; Kugler, Kelso, \& Turvey, 1980). These structures are ensembles of motoric and physiological variables (including muscle movements) that are synergistically related and thus constrained to cooperate or compensate for each other. Thus one set of muscle movements may recruit the action of other synergistically-related muscle movements depending upon the effort exerted by the moving muscle and the relation of its neural control to that of other muscles.

Utilizing this theoretical framework, Michel et al. proposed that the brow movements involved in infant "interest" expressions may be components of larger motoric ensembles involving head and eye movements. Thus, raised brows would tend to occur when infants looked upward, utilizing a complex action pattern involving muscle movements that raise the infant's head and eyes. Similarly, knit brows would tend to occur when infants looked downward, lowering the head and eyes. If such head, gaze, and brow movements occurred accompanied by slightly parted lips, then the infant might be scored as expressing "interest" irrespective of whether one might presume this emotion to be present based on other grounds. Michel et al. proceeded to examine the relationships between infants' head, eye, and brow movements in 5- and 7-month old infants who were presented with toys either above or below eye level. Raised brow movements significantly co-occurred with raised head and eye movements at both ages. Knit brows significantly co-occurred with lowered eyes at 5 months and lowered head at 7 months. Thus raised brows - and to some extent, knit brows — appeared to be part of coordinated muscle ensembles as would be predicted by dynamical systems theory.

Extending this argument to other facial configurations, the "surprise" expression would also seem a likely candidate for reanalysis in terms of dynamical systems theory and possibly a reinterpretation of its emotion status in infancy. As described for both infants and adults (Ekman \& Friesen, 1975; Izard et al., 1983), the complete facial configuration of surprise involves raised brows, raised eyelids, and a moderately open or widely 
opened mouth. According to Izard's AFFEX coding system for infants, if any two of these three facial components are present, then a "surprise" expression may be scored.

Surprise expressions have been conspicuous by their absence in the many infant studies of object permanence that are widely held to elicit surprise in infants (see Camras, Malatesta, \& Izard, 1991, for review). Even with infants as old as 18 months, surprise configurations are rarely observed (Vaughn \& Sroufe, 1976).

Consequently, investigators of infants' object knowledge have been unable to use surprise expressions as their response measure, relying instead on other indices such as gaze behavior.

In contrast to the laboratory studies of object permanence, Camras (1992) often observed "surprise" expressions during a single-infant naturalistic study of her daughter's expressive development during infancy. However, consistent with laboratory studies of object permanence, the infant's "surprise" expressions were not produced in situations that were likely to have elicited surprise. Instead, they appeared to be components of an appetitive response. For example, during the third and fourth months, they appeared when the infant was in a state of excited attention and was reaching toward a desirable but familiar object (e.g., the soft glow of a lamp). By 5 to 7 months, "surprise" configurations were observed to frequently occur as the infant brought an object to her mouth for mouthing.

From the perspective of dynamical systems theory, these observations suggest that the facial actions of mouth opening, brow raising, and possibly upper eyelid lifting comprise a coordinated motor ensemble that sometimes operates as an attentional or appetitive response rather than an expression of the emotion "surprise." This hypothesis is notably similar to Peiper's (1963) proposal that many infant facial expressions occur as "spreading" sensory reactions that increase or decrease receptivity to external stimuli. Peiper specifically noted that infants (and children) may open their mouths during attentive listening or visual observation, representing a spreading orientation response. We propose that such spreading reactions may occur in the reverse direction (from mouth to brows and eyes) and place this phenomenon within the larger theoretical context of dynamical systems theory.

The present study was designed to investigate systematically the hypothesis that mouth opening is selectively accompanied by brow raising and possibly upper eyelid raising in 5- to 7-month-old infants. Towards this end, we examine the co-occurrence of both brow raises and other types of brow movements (i.e., knit brows) with mouth opening. In addition, cooccurrences of mouth opening and upper eyelid raising are examined.

\section{Method}

\section{Participants}

Thirteen healthy infants participated in the study (8 Caucasian-Americans, 2 Hispanic-Americans, 2 AsianAmericans, and 1 African-American). Four infants (3 males) were 5 months of age at the time of testing while nine infants (5 males) were 7 months.

\section{Procedure}

Each infant was seated on the mother's lap facing a small table whose surface was between the infant's chest and navel. The mother was instructed to hold her infant's waist and chest. The infant received 20 midline presentations of toys (10 toys presented once, 5 toys presented twice). For half the presentations, the toy was held slightly above the infant's head and line of vision while for the other presentations, the toys were placed on the table, below the line of sight. The presenter stood in front of the infant but slightly to the right for half the infants and slightly to the left for half the infants in order to avoid blocking the videocamera. The toys included rattles, keys, beads, concentric rings, balls, chains, and cubes. Five toys (cube, beads, chain, key set, concentric circles) were presented twice: once from above and once on the table. The order of presentation was random and the toy was removed 10 seconds after the infant last touched it or 15 seconds after presentation.

The procedure was videorecorded by a Panasonic videocamcorder positioned 2 meters in front of the infant. The videotapes provided a full- face, straight-on view of the infant's face, chest, and arms. 


\section{Coding}

Identification of coding intervals. Each infant's videotape was viewed in order to select two instances of mouth opening to be included in the study. These "target events" were required to meet several criteria. First, the mouth must be opened far enough to be coded as AU 26 (jaw open) or AU 27 (mouth stretch) according to Ekman \& Friesen's Facial Action Coding System (FACS, 1978). Second, no other facial action involving the mouth could be present (thus eliminating, for example, open-mouth smiles). Third, neither the infant's head nor gaze could be lifted 45 degrees or more from the horizontal plane. This last criterion was included because Michel et al.'s (1992) study found that raising the head and gaze tends to recruit brow raising irrespective of mouth position.

The coder viewed the infant's tape starting from the beginning of the experimental procedure. Once the first target event was identified, the coder located the apex of this mouth opening movement. Next the coder identified an interval beginning 2 seconds before the apex of the target movement and ending 2 seconds after the apex. If the infant's head and gaze were not lifted during this time, then the interval was selected as the first target episode to be later coded for accompanying facial movements. If the infant's head and gaze were raised 45 degrees above the horizon at any point within the 4-second interval, then the episode was eliminated. The coder proceeded through the videotape until two target episodes that met all criteria were identified for each infant. For all target episodes, the mouth opening movement began before the onset of the coding interval.

After identifying the target (mouth opening) episodes, the coder selected 2 control episodes to which the target episodes might be compared. The coder searched both before and after each target episode until she could identify the 4-second interval closest to each target that met the following criteria. First, the infant's lips were relaxed and closed or slightly parted during the entire interval. The mouth could not open widely enough to be coded AU 26 or AU 27 as for the target episodes. Second, neither the infant's head nor gaze could be raised 45 degrees or more above the horizontal plane within the 4-second episode. Using this procedure, 2 target (mouth open) episodes and 2 control (mouth closed) episodes were identified for each infant.

Brow, nose, and eyelid movement coding. All brow movements whose apex occurred within a target episode or control episode were coded using FACS criteria. However, the movements also met MAX and AFFEX coding criteria as indicated below. The observed brow movements fell into two coding categories: 1) raised brows, and 2) knit brows. In raised brows, the lateral and medial portion of the brows were raised (FACS AUs $1+2$; MAX Code 20; AFFEX component of surprise). For knit brows, the inner corners of the brows were drawn together and the inner and medial portions of the brows might be lowered (FACS AU 4; MAX Code 20 or 25; AFFEX component of interest or anger).

Nose wrinkling was coded when the skin along the sides of the nose pulled upward, typically causing wrinkling on the sides and/or across the root of the nose (FACS AU 9; MAX Code 42; AFFEX component of disgust).

Upper eyelid raising was also coded using a modification of the FACS criteria for AU 5 (upper lid raiser). Two categories of eyelid raise were used: 1) "trace," i.e., some trace of lid raising is discerned but minimal requirements for FACS coding AU 5 are not met, and 2) slight-to-maximum, i.e., lid is raised enough to meet FACS coding criteria. The FACS coding criteria for AU 5 are as follows: a) if the upper lid normally covers part of the iris in the neutral face, then the upper lid raise must be sufficient to expose virtually the entire iris, or b) if the entire iris shows in the neutral face, the upper lid raise must be sufficient to expose more than a hairline's width of sclera above the iris.

Training and reliability. Both the primary coder and a "reliability" coder were trained in Izard's MAX (Izard, 1979) and AFFEX (Izard et al., 1983) facial coding systems. In addition, the reliability coder was certified to use Ekman and Friesen's (1978) Facial Action Coding System (FACS) and the primary coder was trained to identify the facial movements examined in this study utilizing FACS criteria. 
Both coders scored $12 \%$ of the data for those facial movements examined in the study. Reliability was computed by calculating the number of agreements divided the number of agreements plus disagreements regarding the facial actions present within each 1 -second interval of mutually coded data. Reliability was .75, a level considered acceptable for the coding of facial behavior (Ekman \& Friesen, 1978).

\section{Results}

All infants produced one or more brow movements during their target episodes. In contrast, only one brow raise was produced during the control episodes; 12 of the 13 infants produced zero brow movements during these episodes. Because there was virtually no variance in control episode scores, the data were analyzed utilizing nonparametric tests.

Data analysis indicated that babies produced more brow raises during their target episodes than during their control episodes, sign test, $\mathrm{p}<.003$. However, contrary to our expectations, babies also produced knit brows more often during the target episodes than during the control episodes, sign test, $\mathrm{p}<.04$.

Because previous research (Michel et al., 1992) found some evidence that knit brows are recruited by lowering the head and/or gaze, the occurrence of knit brows with these head and gaze movements was examined. Most of the target episodes (21 of 26) involved lowered head and/or gaze. A Fisher exact probability test failed to show that knit brows occurred more (or less) often in these episodes than in episodes involving only level head and gaze $(\mathrm{p}=.50)$. Furthermore, virtually all control episodes ( 25 of 26$)$ included lowered head and/or gaze, yet no knit brows were observed in these episodes. Thus, the co-occurrence of knit brows and mouth opening could not be attributed to recruitment by lowering the head and/or gaze.

Informal inspection of the videotapes suggested that knit brows were occurring when infants momentarily wrinkled their noses as they mouthed the object. All 5 episodes that included nose wrinkles also included knit brows, accounting for 5 of the 8 knit brow movements that were observed.

The data were thus reanalyzed after omitting all episodes in which nose wrinkling was produced. Results again showed that babies produced brow raises significantly more often during the target episodes than during the control episodes, sign test, $\mathrm{p}<.006$. In contrast, babies were not found to produce knit brows more often in the target episodes, sign test, $\mathrm{p}>.05$. Thus, the mouth-opening movements characteristic of "surprise" expressions (and unaccompanied by momentary nose wrinkling) appeared to be selectively accompanied by raised brows as opposed to other brow movements. Furthermore, all brow raises occurred after the onset of the mouth- opening movement.

No eyelid raises that met minimal FACS criteria were observed. However, 14 of the 26 target episodes included "trace" level eyelid movements. A sign test showed that babies produced more trace eyelid raises during target episodes than control episodes, $\mathrm{p}<.003$. Furthermore, babies produced trace eyelid raises in target episodes involving raised brows more often than in target episodes not involving raised brows, sign test, $\mathrm{p}<.03$. Thus, most trace eyelid raises co-occurred with brow raises.

Formal analyses of age or sex differences were precluded by the small sample size and unequal distribution of infants across sex and age categories. However, inspection of the data suggested that brow raises tended to occur more often in female infants than in male infants (i.e., in $90 \%$ vs. $56 \%$ of their mouth opening episodes). No sex or age trends were noted for eyelid raises.

\section{Discussion}

This study showed that mouth opening tends to be accompanied by brow raising in 5- to 7-month-old infants. Traces of eyelid raising were also observed to occur with many of the brow raises. In contrast, knit brows were not typically observed unless nose wrinkling also occurred. Thus the mouth-opening movements appeared to be selectively associated with raised brows as opposed to other forms of brow activity. In addition, all brow raises 
occurred after the onset of the mouth-opening movement, further suggesting that mouth opening recruits raised brows.

The facial configuration of open mouth and raised brows (with or without raised eyelids) is codable as a "surprise" expression according to both FACS (Ekman et al., 1978) and AFFEX (Izard et al., 1983), a widelyused system for identifying infant emotional expressions. Yet the "surprise" expressions observed in this study occurred in circumstances that would not be expected to elicit surprise in infants. Indeed, the stimulus procedure was actually conducted as part of a larger study of the development of preferential hand use. The procedure involved no sudden, startling, or even unusual objects or activities. Infants were simply handed a set of typical infant toys by an experimenter. Not surprisingly, the infants often brought these objects to their mouths. The mouth openings and brow raises observed in this study usually occurred during this mouthing activity. Thus our results are consistent with Camras' earlier observations of "surprise" expressions produced by her daughter at 5 to 7 months during episodes of object mouthing.

The association of brow raising with mouth opening exemplifies Peiper's (1963) concept of a "spreading reaction." According to Peiper, appetitive or defensive reactions initiated by a sensory organ may often "spread" to other sensory organs. Thus, Peiper described recruitment of mouth opening during visual or auditory attention by infants. Consistent with Peiper, we observed muscle activity in parts of the face peripheral to the instrumentally activated sensory organ. However, while Peiper observed recruitment of mouth opening, in our case, we observed recruitment by mouth opening. The present study together with that of Michel et al. (1992) provide the first systematic experimental demonstrations of this phenomenon.

Our results are also consistent with a dynamical systems perspective on motoric actions. According to this perspective, facial muscle actions may be produced as part of larger "coordinative motor structures" or sets of synergistically related physiological variables, including muscle actions. When one component of the structure is activated, other components may be activated in a cooperative or compensatory relationship. While both Peiper's theory and dynamical systems theory involve similar principles of activation, dynamical systems theory extends these principles beyond the realm of sensory reactions to include synergistic relationships within coordinative structures that may be organized upon some nonsensory basis. Thus the dynamical systems perspective can account for Michel et al.'s finding that raised versus lowered head/gaze movements are accompanied by different brow actions although both types of directional change may occur in the service of the same sensory reaction (visual attention). Because of its broader scope, we prefer to place our findings within the framework of dynamical systems theory.

In the present study, virtually all target episodes involved a fairly extreme widening of the lips (AU 27 rather than AU 26) and occurred as the infants prepared to mouth a toy. Further studies must determine whether our findings would generalize to episodes of mouth opening with parameters differing from those in our study (e.g., less extreme widening of the lips, eliciting contexts that do not involve mouthing objects). In addition, further studies utilizing larger sample sizes must pursue the possibility of significant sex or age differences in the phenomenon. Determining the precise range of circumstances under which recruitment occurs is crucial to understanding the operation of any dynamic system.

While the present study demonstrated that mouth opening was accompanied by brow raising, a dependent relationship in the reverse direction (i.e., brow raising predictably accompanied by mouth opening) was not examined. However, Michel et al. did investigate this possibility. In their study, instances of raised brows were identified and accompanying head, gaze, and facial movements were coded. Relatively few raised brow movements were accompanied by the moderate or wide mouth opening movements investigated in the present study (i.e., FACS AU 26 or 27). This observation indicates that synergistic relationships among components of a coordinative motor structure are not necessarily reciprocal. Furthermore, a muscle action such as raised brows may be a component of more than one coordinative structure and thus may occur with very different sets of muscle actions (e.g., mouth opening or head/gaze lifting). Thus the "meaning" of any particular facial muscle action may depend upon its motor action context as well as its situational context. 
While Michel et al. (1992) did not observe mouth opening to typically accompany raised brows in infants, further investigation might successfully reveal circumstances in which this does happen. Thus, mouth opening might possibly accompany brow raising when the raised brow movement is produced as an instrumental action rather than as a synergistic accompaniment to head/gaze lifting. For example, anecdotal observations suggest that women tend to open their mouths while raising their brows to apply eyeshadow (Feldman, 1986). In addition, as suggested by Camras' (1992) observation of her daughter at 2 to 5 months, mouth opening might occur when infants are in a state of greater excitement and arousal than were subjects in the present investigation. Understanding the complete range of circumstances in which various facial movements are produced is critical to the resolution of issues surrounding the use of facial expression as a measure of infant emotions. With regard to identifying infant emotions, our results indicate that caution should be exercised in using facial expressions as one's sole criteria. The "surprise" expressions we observed were unlikely to reflect the emotion of surprise. Similarly, Michel et al.'s study indicated that "interest" expressions may sometimes be produced by nonemotion causal factors. Thus, researchers might reasonably be enjoined to seek a convergence between facial expressions and judiciously chosen nonfacial indices in the measurement of infant emotions. Such an approach would be consistent with a dynamic systems perspective in which emotions are viewed as sets of interrelated components rather than as single criterion variables.

As indicated earlier, "surprise" expressions are rarely observed in procedures commonly acknowledged to elicit surprise in infants (i.e., object concept studies). Thus facial criteria might be particularly inappropriate for identifying this emotion. Further studies are necessary to determine when the classic facial configuration of surprise becomes associated with surprise as an emotion during the course of development. Such studies might also determine whether the association is an exclusive one.

Evidence reviewed by Camras et al. (1991) suggests that several other discrete emotional expressions identified by the AFFEX system (e.g., "pain," "anger") may also be produced in situations in which these emotions are unlikely to occur. Whereas traditional theories of emotional expression (DET) cannot account for such situational inappropriateness, these observations are compatible with a dynamical systems view. According to dynamical systems theory, coordinative motor structures should be available for recruitment by a variety of systems. Therefore, unique and exclusive ties may never be formed between facial expressions and some discrete emotions.

\section{References}

Barrett, K., \& Campos, J. (1987). Perspectives on emotional development Il: A functionalist approach to emotions. In J. Osofoky (Ed.), Handbook of infant development, (2nd ed.) (pp. 555-578). New York: John Wiley \& Sons.

Bernstein, N. (1967). Coordination and regulation of movements. New York: Pergamon. Buck, R. (1988). Human motivation and emotion (2nd ed.). New York: John Wiley \& Sons. Camras, L. A. (1992). Expressive development and basic emotions. Cognition and Emotion, 6

$(3,4), 269-284$.

Camras, L. A., Holland, E. A., \& Patterson, M. J. (1993). Facial expression. In M. Lewis \& J. Haviland (Eds.), Handbook of emotions (pp. 199-208). New York: Guilford.

Camras, L. A., Malatesta, C., \& Izard, C. (1991). The development of facial expressions in infancy. In R. Feldman and B. Rime (Eds.), Fundamentals of nonverbal behavior (pp. 73105). Cambridge, UK: Cambridge University Press.

Ekman, P. (1972). Universals and cultural differences in facial expressions of emotion. In J. Cole (Ed.), Nebraska Symposium on Motivation, 1971 (pp. 207-283). Lincoln, NE: University of Nebraska Press. Ekman, P. (1982). Emotion in the human face, (2nd ed.). Cambridge, UK: Cambridge University Press. Ekman, R (1989). The argument and evidence about universals in facial expression of emotion. In H. Wagner \& A. Manstead (Eds.), Handbook of social psychophysiology (pp. 143-161). Chichester, England: John Wiley \& Sons. 
Ekman, R (1992). Facial expressions of emotion: New findings, new questions. Psychological Science, 3, 3438.

Ekman, P. (1994). Strong evidence for universals in facial expressions: A reply to Russell's mistaken critique. Psychological Bulletin, 115, 268-287.

Ekman, P., \& Friesen, W. V. (1975). Unmasking the face. Englewood Cliffs, NJ: Prentice-Hall. Ekman, R, \& Friesen, W. (1978). The Facial Action Coding System. Palo Alto, CA: Consulting Psychologists Press.

Emde, R., Gaensbauer, T., \& Harmon, R. J. (1976). Emotional expression in infancy: A bio- behavioral study. Psychological Issues Monographs, 10, 1-189.

Feldman, D. (1986). Imponderables: The solutions to the mysteries of everyday life. New York: Wm. Morrow \& Co.

Fogel, A., \& Thelen, E. (1987). The development of early expressive and communicative action. Developmental Psychology, 23, 747-761.

Izard, C. (1977). Human emotions. New York: Plenum.

Izard, C. (1979). The Maximally Discriminative Facial Movement Coding System (MAX). Newark, DE:

University of Delaware, Instructional Resources Center.

Izard, C. (1991). The psychology of emotions. New York: Plenum.

Izard, C., Dougherty, L., \& Hembree, E. (1983). A system for identifying affect expressions by holistic judgments (AFFEX). Newark, DE: Instructional Resources Center, University of Delaware, Newark, Delaware, 19711.

Izard, C., \& Malatesta, C. (1987). Perspectives on emotional development I: Differential emotions theory of early emotional development. In J. D. Osofsky (Ed.), Handbook of infant development (pp. 494-554). New York: John Wiley \& Sons.

Kelso, J. (1981). Contrasting perspectives on order \& regulations. In J. Long \& A. Baddeley (Eds.), Attention and performance, Vol. 9 (pp. 437-457). Hillsdale, NJ: Erlbaum.

Kelso, J., \& Scholz, J. (1986). Cooperative phenomenon in biological motion. In H. Haken (Ed.), Synergetics of complex systems in physics, chemistry, and biology (pp. 124-149). New York: Springer-Verlag.

Kugler, R, Kelso, J., \& Turvey, M. (1980). On the concept of coordinative structures as dissipative structures: I. Theoretical line. In G. Stelmach \& J. Requin (Eds.), Tutorials in motor behavior (pp. 3-48). Amsterdam: NorthHolland Publishing Co.

Kugler, R, Kelso, J., \& Turvey, M. (1982). On the control and co-ordination of naturally developing systems. In J. Kelso \& J. Clark (Eds.), The development of movement control and co-ordination (pp. 5-78). New York: John Wiley \& Sons.

Lewis, M., \& Michalson, L. (1983). Children's emotions and moods. New York: Plenum. Michel, G. (1991).

Development of infant manual skills: Motor programs, schemata or dy-

namic systems? In J. Fagard \& R Wolff (Eds.), The development of timing control and

temporal organization in coordinated action (pp. 175-199). New York: Elsevier.

Michel, G., Camras, L., \& Sullivan, J. (1992). Infant interest expressions as coordinative motor

structures. Infant Behavior and Development, 15, 347-358.

Oster, H., Hegley, D., \& Nagel, L. (1992). Adult judgments and fine-grained analysis of infant facial expressions. Developmental Psychology, 28, 1115-1131.

Peiper, A. (1963). Cerebral function in infancy and childhood. B. Nagler \& H. Nagler (Trans.). New York:

Consultants Bureau.

Russell, J. A. (1994). Is there universal recognition of emotion from facial expression? A review of the crosscultural studies. Psychological Bulletin, 115, 102-141.

Schoner, G., \& Kelso, J. (1988). Dynamic pattern generation in behavioral and neural systems. Science, 239, 1513-1520.

Sroufe, L. A. (1979). Socioemotional development. In J. Osofsky (Ed.), Handbook of infant development (pp. 462-516). New York: John Wiley \& Sons.

Thelen, E., Kelso, J., \& Fogel, A. (1987). Self-organizing systems and infant motor develop.. ment.

Developmental Review, 7, 39-65.

Thelen, E., \& Ulrich, B. (1991). Hidden skills. Monographs of the Society for Research in Child Development, 56. Whole issue. 
Vaughn, B., \& Sroufe, L. A. (1976). The face of surprise in infants. Paper presented at the meetings of the Animal Behavior Society, Boulder, CO.

Wagner, H., Buck, R., \& Winterbotham, M. (1993). Communication of specific emotions: Gender differences in sending accuracy and communication measures. Journal of Nonverbal Behavior, 17, 29-53. 